\title{
An initial exploration of the perspectives and experiences of diverse learners' acceptance of online educational engineering games as learning tools in the classroom
}

\author{
Kimberly Cook-Chennault \\ Mechanical and Aerospace \\ Engineering Department \\ Rutgers, the State University of \\ New Jersey \\ Piscataway, NJ 08854 \\ cookchen@soe.rutgers.edu
}

\author{
Idalis Villanueva \\ Department of Engineering \\ Education \\ Utah State University \\ Logan, Utah 84322 \\ Idalis.villanueva@usu.edu
}

\begin{abstract}
This Work-In-Progress falls within the research category of study and, focuses on the experiences and perceptions of firstand second year engineering students when using an online engineering game that was designed to enhance understanding of

game as a "learning tool." Results also point to the need to explore how the crediting and assessment of students' performance and learning gains in these types of games could yield inequitable experiences in these types of courses.
\end{abstract} statics concepts. Technology and online games are increasingly being used in engineering education to help students gain competencies in technical domains in the engineering field. Less is known about the way that these online games are designed and incorporated into the classroom environment and how these factors can ignite inequitable perspectives and experiences among engineering students. Also, little if any work that combines the TAM model and intersectionality of race and gender in engineering education has been done, though several studies have been modified to account for gender or race. This study expands upon the Technology Acceptance Model (TAM) by exploring perspectives of intersectional groups (defined as women of color who are engineering students). A Mixed Method Sequential Exploratory Research Design approach was used that extends the TAM model. Students were asked to play the engineering educational game, complete an open-ended questionnaire and then to participate in a focus group. Early findings suggest that while many students were open to learning to use the game and recommended inclusion of online engineering educational games as learning tools in classrooms, only a few indicated that they would use this tool to prepare for exams or technical job interviews. Some of the main themes identified in this study included unintended perpetuation of inequality through bias in favor of students who enjoyed competition-based learning and assessment of knowledge, and bias for students having prior experience in playing online games. Competition-based assessment related to presumed learning of course content enhanced student anxiety and feelings of intimidation and led to some students seeking to "game the game" versus learning the material, in efforts to achieve grade goals. Other students associated use of the game and the classroom weighted grading with intense stress that led them to prematurely stop the use of the engineering tool. Initial findings indicate that both game design and how technology is incorporated into the grading and testing of learning outcomes, influence student perceptions of the technology's usefulness and ultimately the acceptance of the online
Keywords - engineering games, online learning, serious games

\section{INTRODUCTION}

Digital serious games and online learning software have been increasingly incorporated into US classrooms over the last two decades. In particular, educational video games have been studied as mechanisms for enhancing the engagement and performance of undergraduate students (UGs) in spatial learning [1], physics [2], computer science[3], general engineering [4], software and electrical engineering[5], mechanical engineering (ME)[6-8], computer aided design [9], and aerospace engineering [10]. While many studies have explored the efficacy of the games in enhancing overall student performance, few studies have explored how the way the games are incorporated into a class, influences student motivation to learn engineering topics and acceptance of gaming technology as an engineering learning tool in the classroom. Less than a handful of these studies have explored games' appeal, efficacy or UG performance as a function of gender. For example, Joiner et al., [11] incorporated the game Race Academy as a learning tool into a mechanical engineering class comprising 138 UGs (15/138 female) and found that there was no difference in "motivation towards engineering" $(4.2 \pm 0.5$, pre- and post-survey results) or in "perceived engineering competence" $(3.4 \pm 0.7$, pre-survey to $3.3 \pm 0.4$, post-survey) after video game use for female students. Few engineering undergraduate studies examine games' impact as a function of other engineering subgroups, e.g.

$\mathrm{X}$ 
racelethnicity, student age, sexuality, or the intersection of the subgroups.

Race and gender are not mutually exclusively, but rather can intersect in various ways, affecting the experiences of women in multiple settings according the Crenshaw[12, 13]. Crenshaw began to use the term intersectionality to describe the social injustice that African-American women were experiencing because of their dual racial and gender identities. Intersectionality recognizes how anxiety and mental and emotional health problems among individuals who are expected to represent their whole race or gender can be created [14]. One untapped area in engineering education is the role that intersectional experiences play in students' responses to the up-and-coming educational tools that engineering educators are beginning to use and the role they play to engineering formation, especially in real-time.

This Work-In-Progress study explores the use of an online engineering educational game, to elucidate how first and second year engineering students accept this form of educational tool in learning engineering statics concepts. The engineering game used in this study was designed to enhance student's understanding of statics concepts via the design of truss structures. First, students were asked to complete an open-ended questionnaire to understand their experiences with the educational online game. Then, students were invited to participate in a focus group to gather their perspectives and experiences with the game in the context of the engineering course. A diverse population of engineering undergraduate students participated in the study and their perceptions of the game were analyzed as a function of their gender and racial/ethnicity. the following:

The research questions addressed in this work are

1. Was the online engineering game effective as an educational tool (Technology Acceptance Model)?

2. Do students believe engineering learning games for classroom instruction should reflect aspects of their ethnicity or culture?

3. How did playing the game influence students' perceptions of themselves as engineers?

\section{DESCRIPTION OF TH ENGINEERING EDUCATIONAL TOOL}

Thirty-nine undergraduate engineering students (freshman through sophomore level) participated in an oncampus study that focused on an engineering educational game, Build-Truss ${ }^{*}$. This online educational tool emphasizes truss structural stability topics covered in the traditional undergraduate Statics curriculum. The game Build-Truss* was selected for this study because it is presently used as an educational tool in an existing engineering statics course at a tier-1 learning institution in the Eastern region of the United States. Instructors that use this tool in the classroom believe that it can support student learning of engineering statics as a supplement to course materials such as the textbook and inclass lectures. The software used in this study was encouraged and suggested by statics instructors for this course and, approved by the university's Internal Review Board.

The goal of the game Build-Truss is to assist students in developing engineering intuition of truss structure behavior when subjected to loads. The software tool is based on finite strain theory that enables the user to visualize material and geometric nonlinearities and dynamic movement of failed/compromised structures[15]. Users play the game by positioning bars and joints to construct a truss structure that can support an external mass and the weight of the truss structure itself. The structure the player builds must consist of joints and bars, where the bars are connected via the joints. Players are rewarded with nut(s) based on the player's ability to create a structure of minimal weight and structural stability. The number of nuts rewarded to the players is based on the structure's ability to support the given load while minimizing the overall weight of the support structure. Participants move the location of the bars and joints on the screen of the game interface while manipulating the weight of the truss by adjusting the thickness of the bars. Participants visualize the success or failure of their structure in real-time, as the structures visibly collapse or maintain their position once the truss structure is completed. The collapse of the structure is punctuated with clanging sounds associated with the destruction of the structure.

The tool is designed to teach students intuition about the relationship between truss structural design, material and geometric nonlinearities, and dynamic failure [15]. No written clues are provided during the game. Also, there are no instructions or rules furnished in the game interface. Supplemental resources are available as document downloads and videos on the software website and in YouTube videos. Students who reported on their experiences with the online learning tool within the context of a classroom environment (during the focus group discussion) were given one in-class lecture on the operation of the game and interpretation of the game results.

\section{RESEARCH DESIGN}

The goals of this project were to explore 1) the perceived usefulness, ease of use and effectiveness of an online engineering educational tool on a diverse population of first and second year engineering students leveraging the Technology Acceptance Model [16]; 2) whether this population of students expected aspects of their ethnicity or culture to be incorporated into the game; and 3) whether playing the game influenced students' perceptions of themselves as future professional engineers. Towards achieving this goal, a Mixed Method Sequential Exploratory Research Design Method was proposed and approved by the

* Pseudonym used to represent the online engineering educational game used in the study. 


\begin{tabular}{|c|}
\hline Questionnaire Questions \\
\hline $\begin{array}{l}\text { Q1: The engineering concepts presented in the game were } \\
\text { intuitive to me. } \\
\text { - Strongly agree (1), Agree (2), Somewhat agree (3), } \\
\text { Neither agree nor disagree (4), Somewhat disagree } \\
\text { (5), Disagree (6), Strongly disagree (7) }\end{array}$ \\
\hline $\begin{array}{l}\text { Q2: I would recommend that this game be used in } \\
\text { classrooms in the future. } \\
\text { - Strongly agree (1), Agree (2), Somewhat agree (3), } \\
\text { Neither agree nor disagree (4), Somewhat disagree } \\
\text { (5), Disagree (6), Strongly disagree (7) }\end{array}$ \\
\hline $\begin{array}{l}\text { Q3: This game reflected aspects of my culture and/or identity. } \\
\text { - } \quad \text { Strongly agree (1), Agree (2), Somewhat agree (3), } \\
\text { Neither agree nor disagree (4), Somewhat disagree } \\
\text { (5), Disagree (6), Strongly disagree (7) }\end{array}$ \\
\hline
\end{tabular}

Q4: This game highlighted how I can be an important addition to the engineering workforce.

Strongly agree (1), Agree (2), Somewhat agree (3), Neither agree nor disagree (4), Somewhat disagree (5), Disagree (6), Strongly disagree (7)

Q5: I reached a level that adequately represents my abilities as an engineering student.

Strongly agree (1), Agree (2), Somewhat agree (3), Neither agree nor disagree (4), Somewhat disagree (5), Disagree (6), Strongly disagree (7)

Q6: This game helped me view myself as an engineer. Strongly agree (1), Agree (2), Somewhat agree (3), Neither agree nor disagree (4), Somewhat disagree (5), Disagree (6), Strongly disagree (7)

\begin{tabular}{l}
\hline Examples of Focus Group Questions \\
\hline Did you enjoy playing the learning game? Explain. \\
\hline I think engineering learning tools should reflect aspects of my \\
culture and/or identity. Explain. \\
\hline Do you play video games on your computer? If you do, what \\
games do you play and why? If not, why not?
\end{tabular}

Institutional Review Board at Rutgers, the State University of New Jersey. The data described herein is the work-inprogress of the first phase of a multiple-year study. All participants in the study were undergraduate engineering students from the School of Engineering. Students provided demographical information such as age range, gender, sexual orientation, ethnicity, undergraduate major and experience with online learning tools in an online questionnaire that was completed as part of the research study. The demographics of the student population studied are provided in Figure 1, where $54 \%$ and $46 \%$ of the respondents were male and female, respectively. This demographic information was correlated to questionnaire questions. Thirty-nine students participated in the study.

The students first played the engineering game for 20 minutes, completed a questionnaire (questions provided in TABLE 1) and then participated in a focus group discussion for $\sim 1$ to 1.5 hours. The questionnaire included Likert- scaled questions pertaining to their experiences with the game, demographical student information and previous experiences with playing video games. The Likert-scale ranges included: Strongly Agree (1), Agree (2), Somewhat Agree (3), Neither Agree nor Disagree (4), Somewhat Disagree (5), Disagree (6) and Strongly Disagree (7), where Strongly Agree and Strongly Disagree were ranked 1 and 7, respectively. During the focus group, participants discussed their perceptions of the game as an engineering educational learning and motivational tool. Questionnaire questions were repeated during the focus group, which enabled a more in depth discussion of the topics described in the Technology

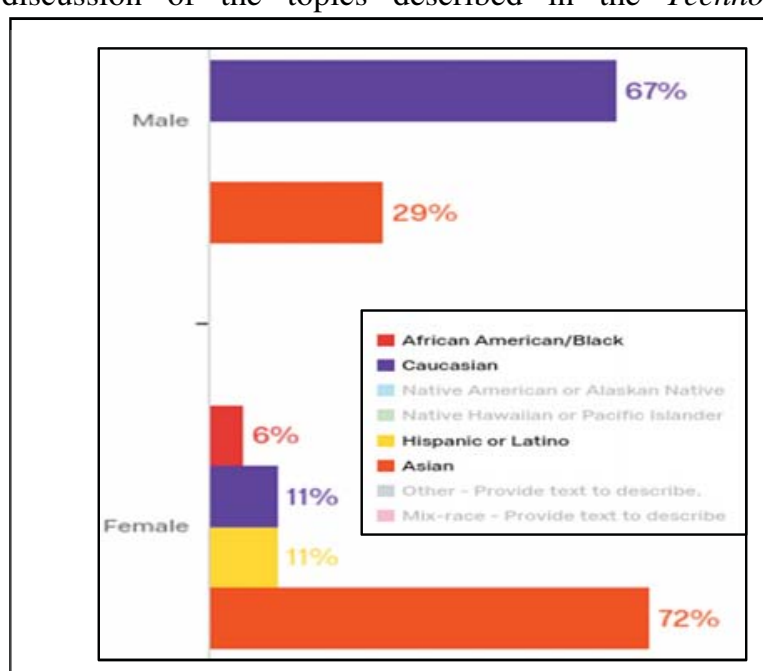

Figure 1: Race and ethnicity demographics of the students.

Acceptance Model (TAM) [16], i.e. perceived usefulness and ease-of-use of the game.

Several additional questions were included along with the questionnaire questions during the focus group discussion to facilitate the exploration of student's opinions regarding their prior experiences with video games, enjoyment playing the Build-Truss game and whether their ethnicity or culture should be included in online engineering educational learning games. Students provided explanations/rational for specific responses to the questionnaire questions and additional information regarding prior experiences with video and educational games/tools within a classroom setting and outside of classroom setting during the focus group discussion. Focus group participants were assigned into groups based on gender and availability of schedule date/time, where each group consisted of $4-6$ participants. Examples of the questionnaire and focus group questions are provided in TABLE 1. All questionnaire questions were examined as a function of race/ethnicity and gender with the aim of elucidating differences in trends associated with student perception as a function of these groups. An open coding approach based on the Grounded Theory Method [17] was employed to categorize similar statements, opinions and experiences discussed during the focus group.

\section{RESULTS AND DISCUSSION}

Thirty-nine undergraduate engineering students participated in this study. The data is presented as a function of gender and race/ethnicity to explore the perceptions of intersectional groups' perceptions and experiences of 
engineering educational serious games. A questionnaire was developed to address aspects of each of the research questions. The responses of this population to an online engineering education game was recorded in Qualtrics and a follow-up focus group was held to understand student's experiences and observations regarding the game. Salient points made during the focus group discussions were grouped into categories based on repeated phrases and wording from transcripts of the focus group discussions.

A. Research Questions 1: Was the online engineering game effective as an educational tool (Technology Acceptance Model)[16]?

Questions 1 and 2 from the questionnaire were motivated by the Technology Acceptance Model developed

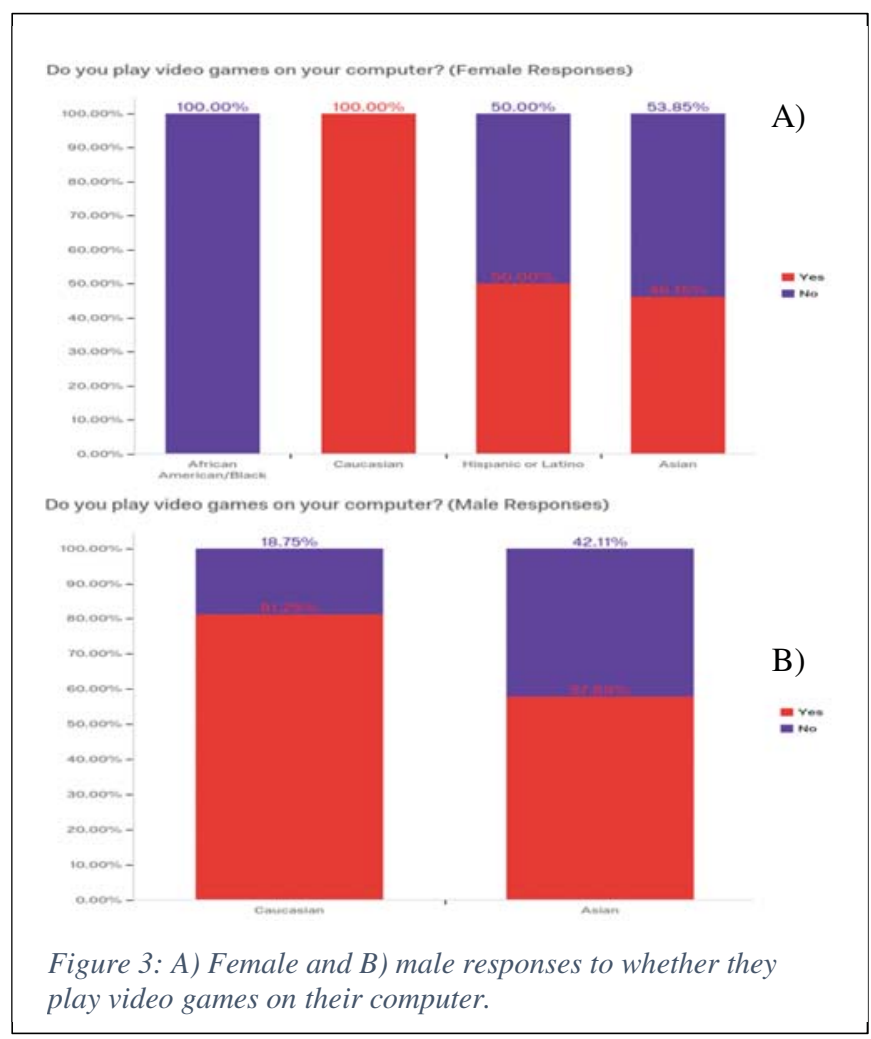

by Davis [16]. According to this educational model, positive attitudes towards using an electronic based system influences an individual's tendency to use the system, and the intention of using an electronic system is a function of an individual's perceived usefulness and perceived ease of use of the electronic system. In Question 1, students were asked if the engineering concepts presented in the game were intuitive. The results from this question are displayed in Figure 2. Their responses were quantified on a scale of Strongly Agree, ranked -1 through Strongly Disagree ranked - 7. Over 50\% and $100 \%$ of the women of color and Caucasian women, respectively either strongly agreed or agreed that the game was intuitively easy to play. Similarly, high percentages of the male respondents agreed that the game was relatively easy to learn to play. However, all respondents indicated during the focus group discussion that there was a distinctive difference between the game being easy to learn to play in terms of building a structure using the user interface; and the game's ability to introduce and teach engineering concepts to be intuitive. Though there was a high percentage of students who indicated that they easily learned how to use the game, many found that they were unaware of or did not fully understand key aspects of the game that were critical to gaining engineering insight into truss design. For example, several students did not realize that the number of nuts rewarded at the end of a challenge was related to the quality of truss designed by them and that optimal designs were those that weighed the least and were able to withstand a predetermined load on the truss ( 7 respondents). Other students were unable to identify the color coding of the truss bars, which indicated whether bars were either in tension or compression. Students who later discovered their "lack" of understanding of the game rules during the focus group were also those who played video games the least in their spare time and had the least exposure to engineering learning games prior to college. The populations of students who played video games the least in their spare time were women of color (Figure 3). Specifically, Caucasian males (83\%) and

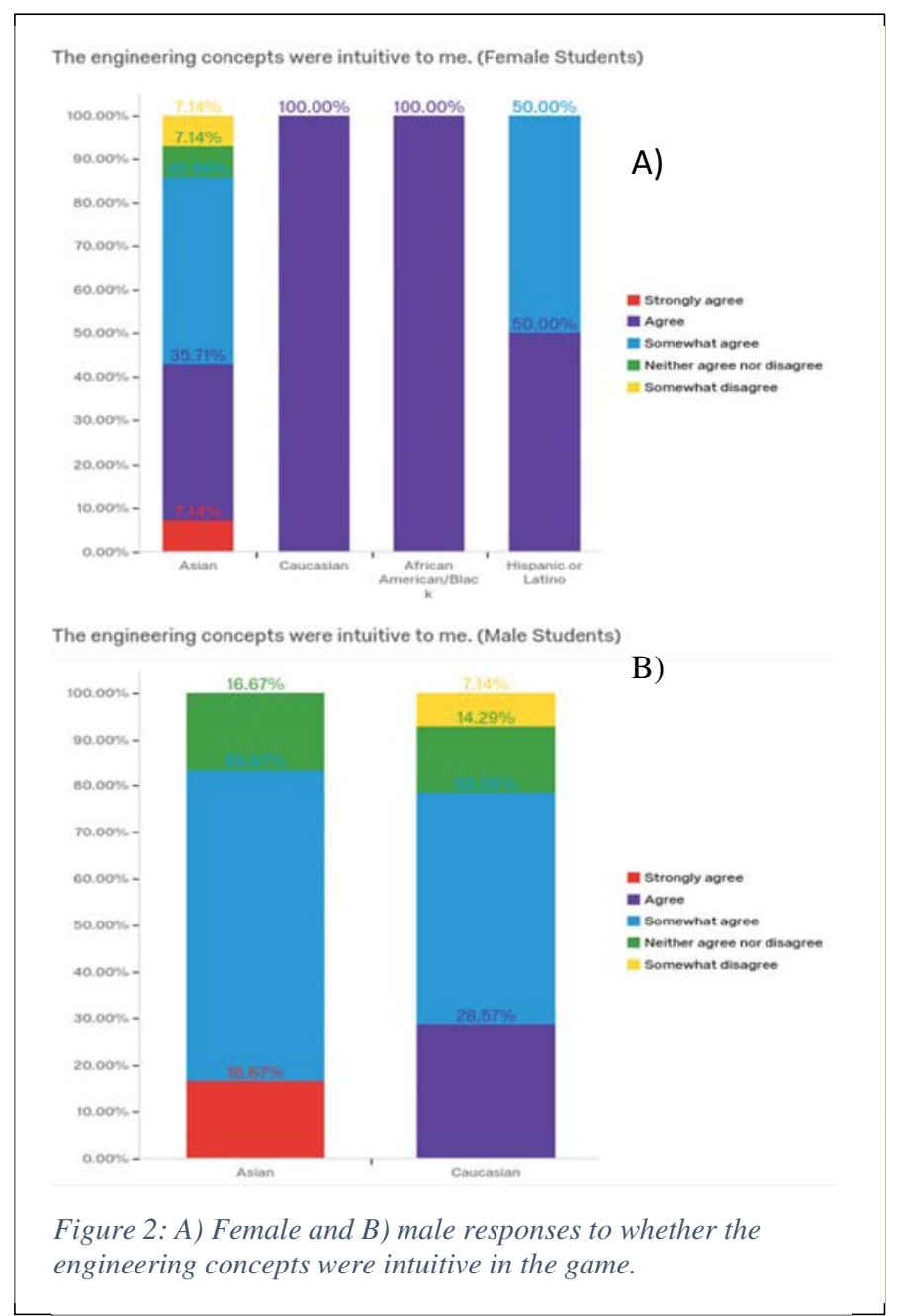


females (100\%) in this study played video games and engineering and physics-based games on their computer more than women of color, i.e. African American (0\%), Latina $(50 \%)$ and Asian $(46 \%)$. Caucasian and Asian male students and one female indicated that they had been exposed to "engineering learning games" through prior middle and high school STEM classes and/or extracurricular clubs/activities, in addition to their playing games on their computer for fun.

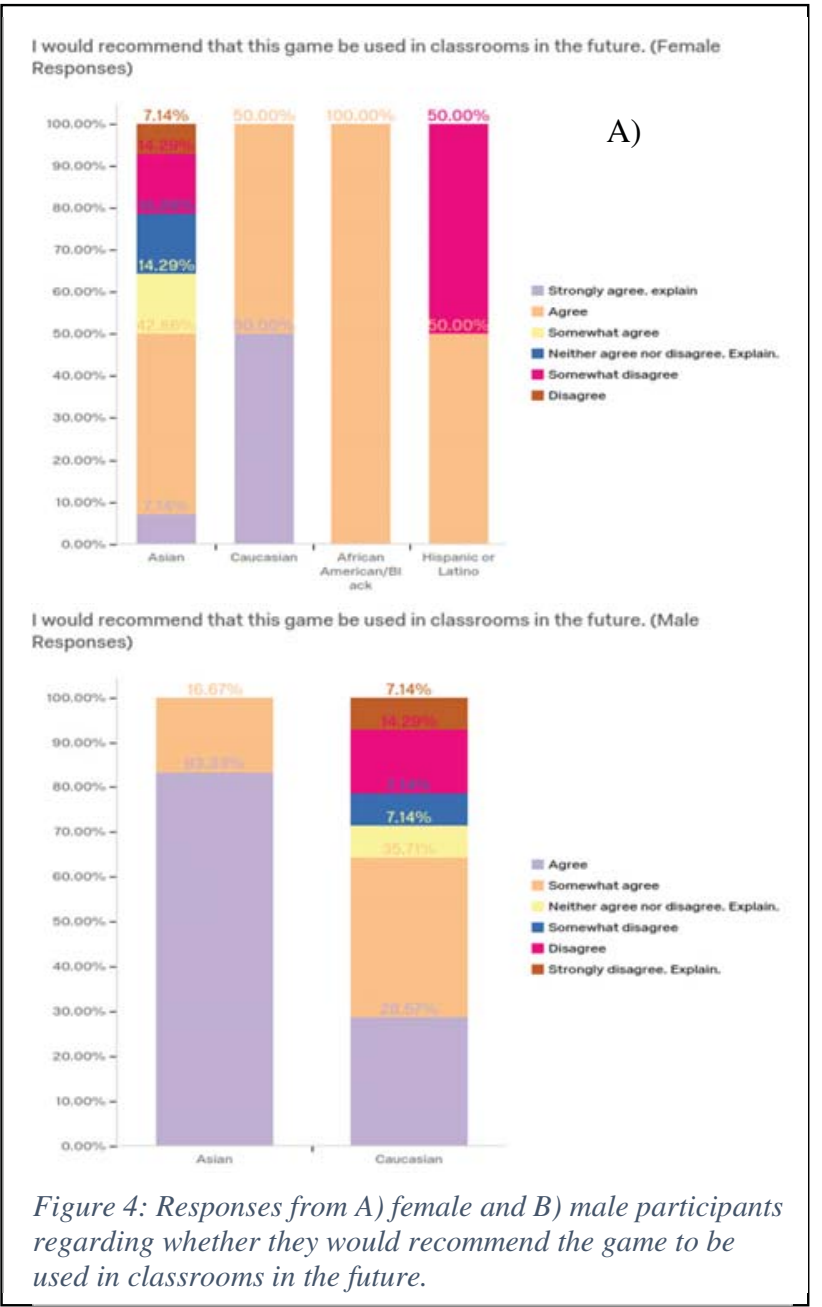

These facts may have contributed to slightly lower recommendations by women of color (50\% Asian and 50\% Latina women) for this game be used in classrooms in the future as shown in Figure 4. In addition, only 2 of the 39 participants indicated that they would use such a tool to prepare for their exams or technical job interviews. It is unclear if limited access and/or knowledge of engineeringbased online learning tools in middle or high schools played a significant role some students' experiences with and acceptance of this this game as a learning tool. It is also unclear if what students perceived as trial and error was in fact the development of their engineering intuition.

Student focus group responses regarding the TAM's [16] perceived usefulness of the game were categorized into two primary responses: 1) the game affirmed coursework at the lowest two or three game challenge levels (20 statements) and 2) engineering intuition from the game broke down at higher Build-Truss challenge levels (20 statements). The game includes 24 Challenge levels. The first three levels may be solved using rudimentary skills gleaned in Statics pertaining to two-dimensional simple truss structures. The game did not provide feedback (oral or text) on failed structures other than loud clanging sounds after structures broke, i.e. the engineering tool simulated structures that separated and broke apart when the structure drawn by the student did not support the load. For example, one student stated, "I wish there was like an instruction box or something to tell you what the point of the game was." As a result, students resorted to creating structures that were successful through trial and error (met game requirements), and not based on engineering skills learned in class (15 statements). This diminished student's ability to directly correlate engineering concepts from the class or textbook with the game and therefore diminished student's perception of the tool as being useful within the classroom environment. In addition, majority of the students were not convinced that the game was teaching them the topics it was designed to cover in statics. For example, students attributed their success in the game to learning how to "game the game" versus their development of engineering skills in cases where students reached high challenge levels (10 stated during focus group discussion). In addition, 9 out of the 39 participants asserted that some people without an engineering background at all would be able to achieve some level of success in the game by trial and error (8 participants out of 39), which lead them to perceive the tool was not useful in learning or validating engineering concepts beyond the first three challenge levels in the game. Another student noted, "...towards the end of the high-level challenges, the solutions... they seemed to make less sense... sometimes I got more nuts and bolts where I felt like my [design] was unrealistic."

Students were also asked during the focus group discussions if they enjoyed playing the engineering game and if they would recommend that this game be used in engineering classrooms in the future. The results are provided in Figure 4, where $50 \%$ or greater of all respondents indicated that they enjoyed playing the game and either agreed or strongly agreed that they would recommend that the game be used in classrooms in the future. Students' responses were also categorized based on their previous experiences with the software being incorporated into a classroom environment. The most prevalent response categories were: 1) post-traumatic stress based on the implementation of the software into the class, 2) demotivation towards using the software based on how the software was implemented into the class and 3) enjoyment of playing the game in the study (20 minutes) versus the classroom environment. Students indicated that that when the software was implemented into their class, it was included as extra credit towards a homework assignment grade. Extra credit could only be earned based winning a competition among students who achieved the most nuts and completed the most difficult game challenges. Several students stated 
that beyond the entry level challenges, they often were unable to easily link their engineering coursework and skills directly to the game. Hence, some students sought solutions from external resources (cheated) to game the game versus learning the engineering material in efforts to achieve grade goals. Other students who did not like video games and/or did not like the competition for obtaining extra credit, associated the competition and the game with intense stress (10 responses). The stressed students decided not to participate in the extra credit or play the game at all with some noting that they saw more benefit in studying from the book for exams and homework than playing a game (5 responses). Hence, making the game and the associated extra credit a competition motivated some students and demotivated others. More women opted to discontinue playing the game and therefore pursuing the extra credit, while majority of the men persisted with the extra credit format. Thus, extra credit linked to competition-based weighting of grades, though unintended, may have created a bias towards more competitively motivated students and/or students who were more comfortable with gaming technology. In addition, the competitive nature of how the extra credit was applied to student grades demotivated student learning as evidenced by student cheating to gain extra credit points. Many respondents stated that while they supported any additional educational tool, it would be most beneficial if incorporated "in the right way" (10 responses). To many students "the right way" meant inclusion of verbal or written hints and game goals to relate the game to class engineering content and a more appealing and realistic looking game interface. Majority of the students explained that inclusion of online software and simulation tools enable them to visualize concepts that were difficult to imagine from the textbook. They deemed the game misses and opportunity to link what was simulated to factual engineering theory. The intuition skills gleaned by students who played the game were not clearly linked to homework and exams and therefore the benefits of the game's usefulness in the classroom setting may not have been clear to many students. Other students indicated that they made a conscious decision "not to cheat" and ultimately, "not to win extra credit while playing the game." These students indicated that they gained profound insight from the time spent playing the game in being able to look at truss structures and intuitively know which members were in tension or compression (3 responses).

B. Research Question 2: Do students believe engineering learning games for classroom instruction should reflect aspects of their ethnicity or culture?

Two questionnaire questions were posed to answer this research question: 1) This game reflected aspects of my culture and/or identity and 2) I think engineering learning tools should reflect aspects of my culture and/or identity. The former questionnaire statement was assessed according to Likert scale, where responses could range from Strongly Agree to Strongly Disagree. The latter statement allowed students to select from three choices: "yes", "maybe" or "no". Majority of the female and male respondents indicated that the truss game did not reflect aspects of their culture and/or identity as shown in Figure 5. On the other hand, majority of the male (over 50\% non-Caucasian and 64\% Caucasian) respondents indicated that the engineering game should not reflect aspects of their culture and identity, while higher percentages of the female respondents (over 60\% nonCaucasian and 50\% Caucasian) selected "maybe". The focus group discussion confirmed the sentiment of this questionnaire statement, wherein female students expressed their openness to inclusion of culture and identity that could enhance the game through storyline and themes. However, due to the small sampling of students and under-represented

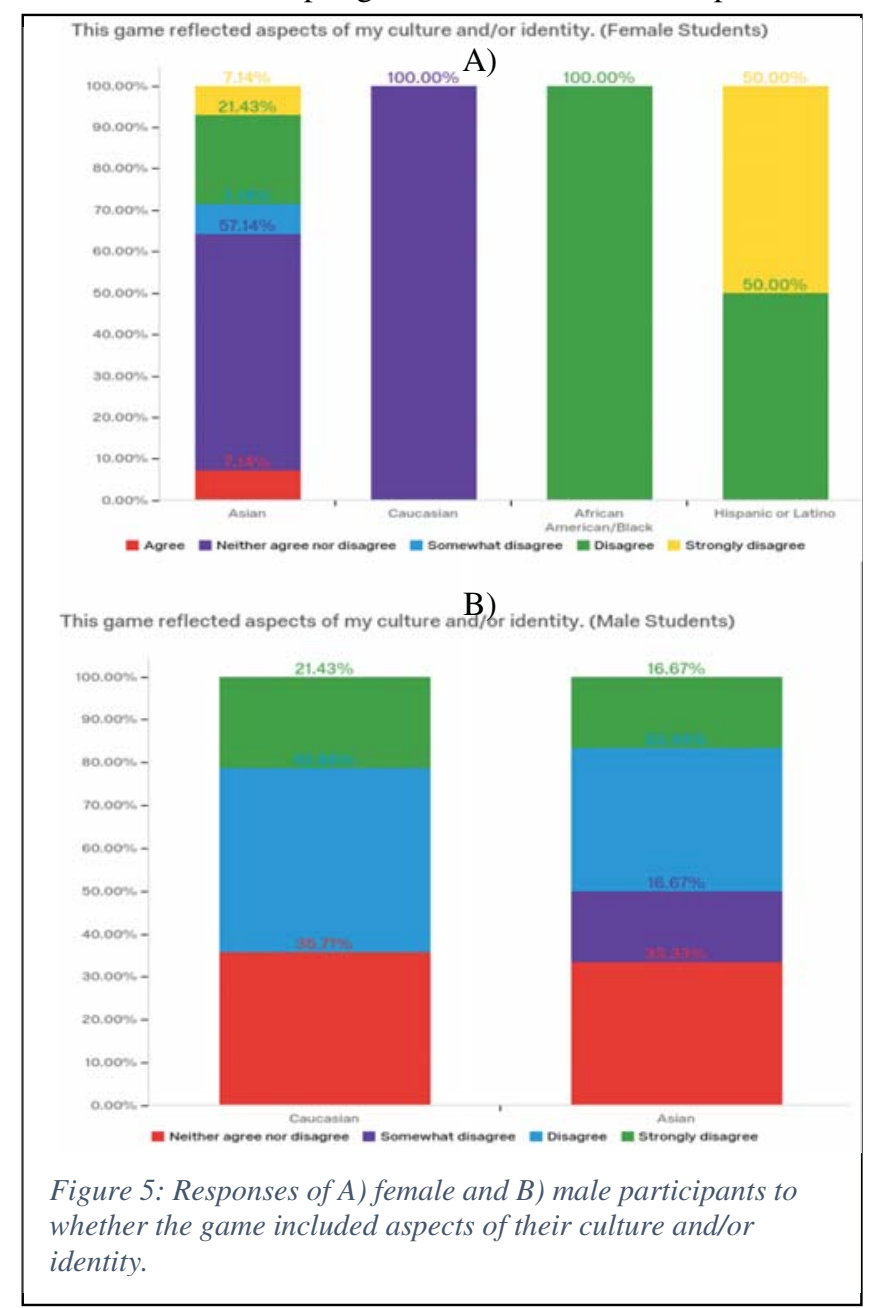

minority groups within the study, a conclusion pertaining to differences in preference for inclusion of gender and race/ethnicity in serious engineering games is inconclusive. 
However, the focus group discussion elucidated key aspects of the respondent's interpretation of the terms culture and identity and whether it is beneficial in their learning condition. First, majority of the respondents correlated the terms "culture and identity" with "race and ethnicity" in their interpretation of this question. This was evidenced by respondent statements (agreed upon by - 5 respondents) such as, "naming a game avatar 'Ravi' or 'Patel' would not enhance my learning of engineering concepts." Second, students indicated that inclusion of stories lines that illustrate the engineering aspects of design, real-world images and realistic looking structures would have enhanced the appeal of the game (7 statements). Though only $16 \%$ of the students stated that inclusion of culture and ethnicity should be embedded in the games, students stated that inclusion of realworld challenges and story lines and images would enhance the game. Real-world game challenges and story lines theoretically embedded cultural elements though students during the discussion dissociated these aspects of cultural context with "culture and identity". Interestingly, the types of real-world examples posed by students during the focus group varied by gender. For example, space flight structures and loading induced by cars travelling over bridges were typical examples given by male respondents, while bridges constructed to transport food and supplies to impoverished people was given by a female respondent. More rigorous investigation to elucidate whether these differences in story line themes resonate along gender and/or cultural/racial lines is needed.

Several of the female respondents indicated that they "did not expect inclusion" (5 statements) of one's culture or identity in an engineering game and thought "it might be nice" or "more interesting" if the games did. Male respondents either dismissed the question in the focus group (did not respond to the question) or were more decidedly opposed to inclusion of culture or ethnicity into engineering games. For example, some students stated (with group agreement) that inclusion of these elements into games would have a "reverse effect" of "turning off students" who were already engaged in engineering ( 3 respondents). Other males commented that inclusion of cultural and identity elements in an engineering game would not necessarily make the engineering content easier to learn or more engaging (2 respondents).

\section{Research Question 3: How did playing the game influence students' perceptions of themselves as engineers?}

Dowling [18, 19] described the formation of engineers as being dictated by formal education and informal training, while others such as Lichtenstein, et al. [20, 21] have acknowledged that persistence in engineering as a student and professional is influenced by one's self identification with their profession. For example, [22] found that students' reaffirmed their identity as engineers when exposed to challenging learning opportunities that they deemed to be positive. Similarly, negative student experiences were also determined to be formative by [22] in perpetuating attitudes of "non-identification" as an engineer and therefore, dissuaded students from future engineering related career plans. Hence, the questionnaire questions that address this topic corresponded to questions 4 through 6 from TABLE 1 . The plotted results are for whether students deemed the game as a tool that highlighted their important addition to the

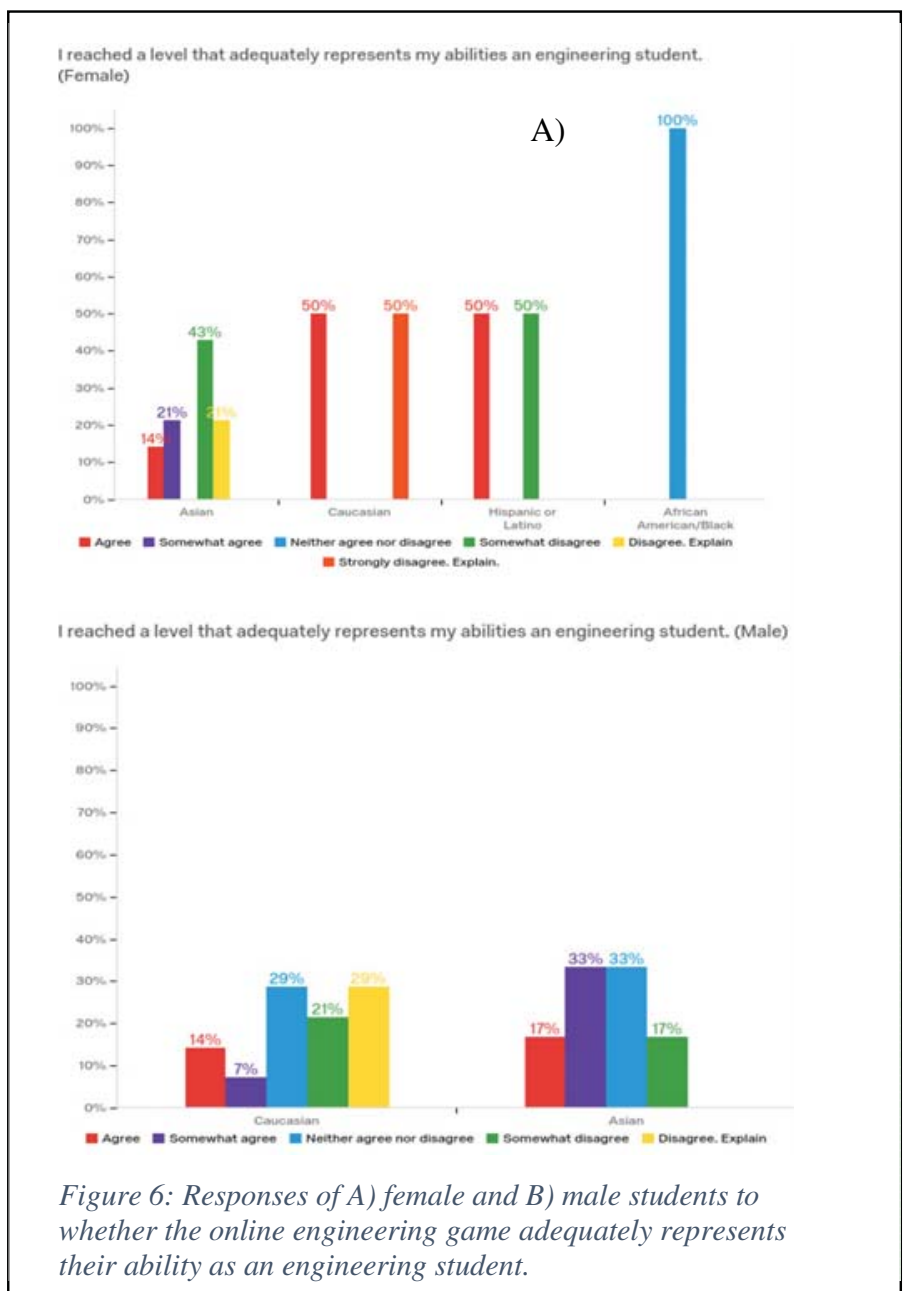

engineering workforce is provided in Figure 7. The results indicate that a higher percentage of female non-Caucasian respondents believed the game illustrated how they could be an important addition to the workforce in comparison to $50 \%$ of Caucasian male and female students who responded that they either strongly or somewhat disagreed or disagreed with this statement. Majority of the female students and Caucasian males agreed that the game did not reflect their abilities as an engineering student (Figure 6), while $50 \%$ of Asian male students did.

In general, students stated that while they knew the game success was not a predictor of their engineering abilities, they equated the fact that they were playing a game that focused on engineering skills, with being indicative of being an engineer ( 9 statements), i.e. viewing themselves as having interests in engineering like activities as shown in Figure 7. On the other hand, students noted that they felt 
frustrated and were intimidated when they were not able to "figure the game out" (6 statements), i.e. not able to directly relate course content with successful or not successful truss structures. Three female students noted that they found it peculiar that the game made "clanking sounds" when their structures failed but, did not offer pleasing or affirmative sounds when they were successful. Female students indicated that affirmative sounds/words, e.g. "Yeah!" or the sound of applause would enhance their feelings of accomplishment when playing the game, while male respondents did not indicate the desire to be positively affirmed while they played the game. Both male and female students provided negative feedback regarding the clanking

This game highlighted how I can be an important addition to the engineering workforce. This game hightighted
(Female Students)

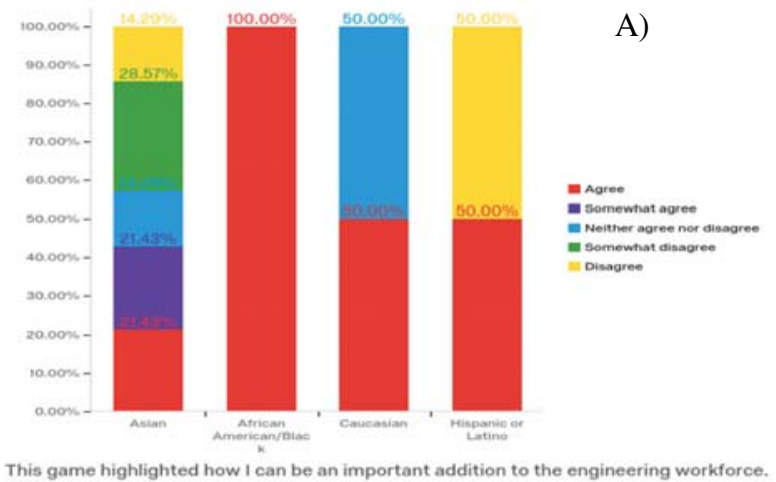

This game highlight (Male Students)

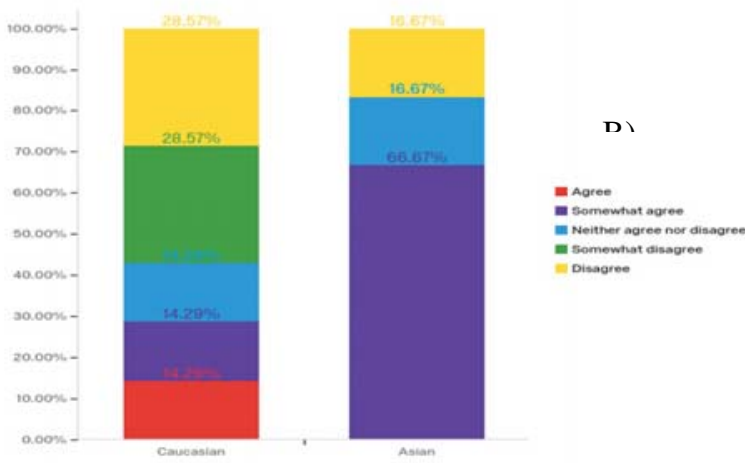

Figure 7: Responses of A) female and B) male students regarding whether the game highlighted how they could be an addition to the engineering workforce.

sounds of the game (14 responses). Men and women associated negative sounds from failed structures with failure in the game and instances of feeling demotivated in their abilities in mastering the course content. Structures that failed the loading conditions crashed (visibly on the screen) and a clanging crash sound accompanied the crash (auditory sound). Many respondents stated that the clanging sounds associated with failed attempts heightened their feelings of frustration with not being able to master a certain level of the game ( 3 out of the 8 who cited sound). They stated this was heightened by their lowered ranking in the competitive grading structure of the class extra credit.

Student focus group responses regarding frustration level was attributed to three primary themes: 1) lack of instruction or hints to indicate rational for failed engineering structure (stated 32 times in focus group); 2) successful designs that rendered more nuts but were unrealistic/unsafe (stated 6 times in focus group) and 3) game design that limited ease of drawing design structures on the screen (stated 10 times during focus group). Of these themes, students expressed concern over an engineering tools that did not directly engage the student with guidance while playing. Students linked these categories to feelings of being demotivated to engage further with the game (10 times in the focus group discussions).

\section{CONCLUSIONS}

This work-in-progress summarizes the findings of a small sample size of participants (39) regarding their assessment of an engineering education tool for gaining intuition on engineering statics of truss structures. Both the focus group discussion and questionnaire were used to glean preliminary answers to the research questions. The responses obtained will be used to modify the questionnaire and design method to better understand the rationale behind responses pertaining to the formation of engineers, inclusion of culture or identity into serious engineering educational online games and acceptance of serious games as engineering educational tools for classroom environments. These preliminary findings suggest that students expect reinforcement and introduction of technical content when using video and serious games as educational tools. In addition, preliminary findings suggest that inquiry-based instruction is most effective when incorporated with a multifaceted schema of tools, e.g. additional sources of information, feedback on successful and failed attempts, opportunities to review evidence, provision of explanations to explain predictions and communication of results and findings. Preliminary results also indicate that the way in which an instructor incorporates the educational tool within the classroom influences how students perceive its usefulness and enjoyment. Weighted grading based on competition for extra credit may introduce bias in acceptance of the education tool wherein those with experience with video game technology perform and engage more readily. Also, bias against students who are not motivated via competition based educational structures may feel discouraged from participating in specific learning opportunities that may benefit them. Furthermore, our findings suggest students have different expectations of engineering educational serious games versus games that are designed for entertainment. Finally, game intuition that is not directly linked to course assessment (exams, etc.) are less 
accepted technologies as those that do. Insights such as these will be used to further develop and modify the questionnaire.

\section{REFERENCES}

[1] J. Martin-Gutierrez, J. L. Saorin, N. Martin-Dorta, and M. Contero, "Do Video Games Improve Spatial Abilities of Engineering Students?," International Journal of Engineering Education, vol. 25, no. 6, pp. 1194-1204, 2009.

[2] D. M. Adams, C. Pilegard, and R. E. Mayer, "Evaluating the Cognitive Consequences of Playing Portal for a Short Duration," Journal of Educational Computing Research, vol. 54, no. 2, pp. 173-195, Apr 2016.

[3] J. I. Alonso, L. de la Ossa, M. J. Flores, J. A. Gamez, J. Martinez-Gomez, and J. M. Puerta, "SUPPORTING INTELLIGENT SYSTEMS TEACHING BY MEANS OF COMPUTER GAMES PROGRAMMING," in Edulearn10: International Conference on Education and New Learning Technologies, L. G. Chova, D. M. Belenguer, and I. C. Torres, Eds. (EDULEARN Proceedings, 2010.

[4] T. A. Philpot, R. H. Hall, N. Hubing, and R. E. Flori, "Using games to teach statics calculation procedures: Application and assessment," (in English), Computer Applications in Engineering Education, Article vol. 13, no. 3, pp. 222-232, Oct 2005.

[5] M. Pantoja, "Designing a New Video Game App as an aid for Introduction to Programming classes that use C Programming Language," Proceedings of the Head'17 - 3rd International Conference on Higher Education Advances, pp. 10-16, 2017.

[6] B. D. Coller and M. J. Scott, "Effectiveness of using a video game to teach a course in mechanical engineering," Computers \& Education, vol. 53, no. 3, pp. 900-912, Nov 2009.

[7] B. D. Coller and Ieee, "Work in Progress - A Video Game for Teaching Dynamics," in 2011 Frontiers in Education Conference(Frontiers in Education Conference, 2011.

[8] B. D. Coller and Ieee, "A Video Game for Teaching Dynamic Systems \& Control to Mechanical Engineering Undergraduates," in 2010 American Control Conference (Proceedings of the American Control Conference, 2010, pp. 390-395.

[9] Z. Kosmadoudi, T. Lim, J. Ritchie, S. Louchart, Y. Liu, and R. Sung, "Engineering design using gameenhanced CAD: The potential to augment the user experience with game elements," Computer-Aided Design, vol. 45, no. 3, pp. 777-795, Mar 2013.

[10] M. Okutsu, D. DeLaurentis, S. Brophy, and J. Lambert, "Teaching an aerospace engineering design course via virtual worlds: A comparative assessment of learning outcomes," Computers \& Education, vol. 60, no. 1, pp. 288-298, Jan 2013.

[11] R. Joiner et al., "Digital Games, Gender and Learning in Engineering: Do Females Benefit as Much as Males?," Journal of Science Education and Technology, vol. 20, no. 2, p. page 182, Apr 2011.
[12] K. Crenshaw, "Demarginalizing the Intersection of Race and Sex: A Black Feminist Critique of Antidiscrimination Doctrine, Feminist Theory and Antiracist Politics," The University of Chicago Legal Forum, vol. 140, pp. 139-167, 1989.

[13] K. Crenshaw, MAPPING THE MARGINS INTERSECTIONALITY, IDENTITY POLITICS, AND VIOLENCE AGAINST WOMEN OF COLOR

(Stanford Law Review Vol 43, No 6, July 1991: In Memoriam: Carl Bernhardt Spaeth; Women of Color at the Center). 1993, pp. 1241-1299.

[14] J. Mens-Verhulst and L. Radtke, "Intersectionality and Mental Health: A Case Study," IST-Travelling, pp. 1-19Available: Last accessed: 02/20/2018

https://static1.squarespace.com/static/56fd7e0bf699bb7d0d3ff8 2d/t/593ab6522e69cf01a44b14d4/1497019986918/IN TERSECTIONALITY+AND+MENTAL+HEALTH2 .pdf

[15] Available:

http://www.scientificmonkey.com/software.html

[16] F. D. Davis, "PERCEIVED USEFULNESS PERCEIVED EASE OF USE, AND USER ACCEPTANCE OF INFORMATION TECHNOLOGY," Mis Quarterly, vol. 13, no. 3, pp. 319-340, Sep 1989.

[17] J. M. Case and G. Light, "Framing Qualitative Methods in Engineering Education Research," in The Cambridge Handbook of Engineering Education Research and Reflections on the Future of the Field. Journal of Engineering Education, A. Johri and B. M. Olds, Eds. New York, NY: Cambridge University Press, 2014, pp. 535 - 549.

[18] G. L. Downey, The Normative Contents of Engineering Formation Engineering Studies (Cambridge Handbook of Engineering Education Research). 2014, pp. 693-711.

[19] G. L. Downey, "Opening up engineering formation," Engineering Studies, vol. 7, no. 2-3, pp. 217-220, Sep 2015.

[20] G. Lichtenstein, H. L. Chen, K. A. Smith, and T. A. Maldonado, Retention and Persistence of Women and Minorities Along the Engineering Pathway in the United States (Cambridge Handbook of Engineering Education Research). 2014, pp. 311-334.

[21] G. Lichtenstein, H. G. Loshbaugh, B. Claar, H. L. Chen, K. Jackson, and S. D. Sheppard, "An Engineering Major Does Not (Necessarily) an Engineer Make: Career Decision Making Among Undergraduate Engineering Majors," Journal of Engineering Education, vol. 98, no. 3, pp. 227-234, Jul 2009.

[22] K. L. Meyers, M. W. Ohland, A. L. Pawley, and C. D. Christopherson, "The Importance of Formative Experiences for Engineering Student Identity," International Journal of Engineering Education, vol. 26, no. 6, pp. 1550-1560, 2010. 